\section{Original Research}

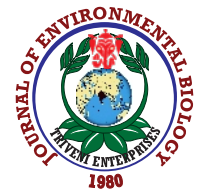

DOI : http://doi.org/10.22438/jeb/41/5/MRN-1317

\title{
Bio-characterizations of some marine bacterial strains isolated from mangrove sediment samples of four major cities of Saudi Arabia
}

\author{
K.A. Qureshi ${ }^{1,2 *}$, M. Seroor', A. Al-Masabi', M.A. Saykhan', Y.A. Mutairi', G.O. Elhassan ${ }^{1}$ and R.A. Khan ${ }^{3}$ \\ 'Department of Pharmaceutics, Unaizah College of Pharmacy (Qassim University), Unaizah, 51911, Saudi Arabia \\ ${ }^{2}$ Department of Biosciences and Biotechnology, Invertis University, Bareilly-243 123, India \\ ${ }^{3}$ Department of Medicinal Chemistry and Pharmacognosy, College of Pharmacy (Qassim University), Buraidah, 51411, Saudi Arabia \\ *Corresponding Author Email : ka.afrah@gmail.com
}

\section{Abstract}

Aim: To isolate, purify and bio-characterize native antibiotic-producing bacterial strains present in the mangrove sediment samples sourced from the coastal areas of four major cities, i.e., Jeddah, Yanbu, Dammam and Jubail of SaudiArabia.

Methodology: The mangrove sediment samples were collected and processed according to the reported standard techniques. Selective Millipore $\circledast$ filter technique was followed to isolate bioactive marine organisms. Isolates were identified by the next-generation $16 \mathrm{~S}$ rRNA sequencing method. The isolated strains were screened for their antimicrobial activity by spot inoculation method. The effects of $\mathrm{pH}$, temperature, and salt concentrations on the growth of isolated bacterial strains were also recorded.

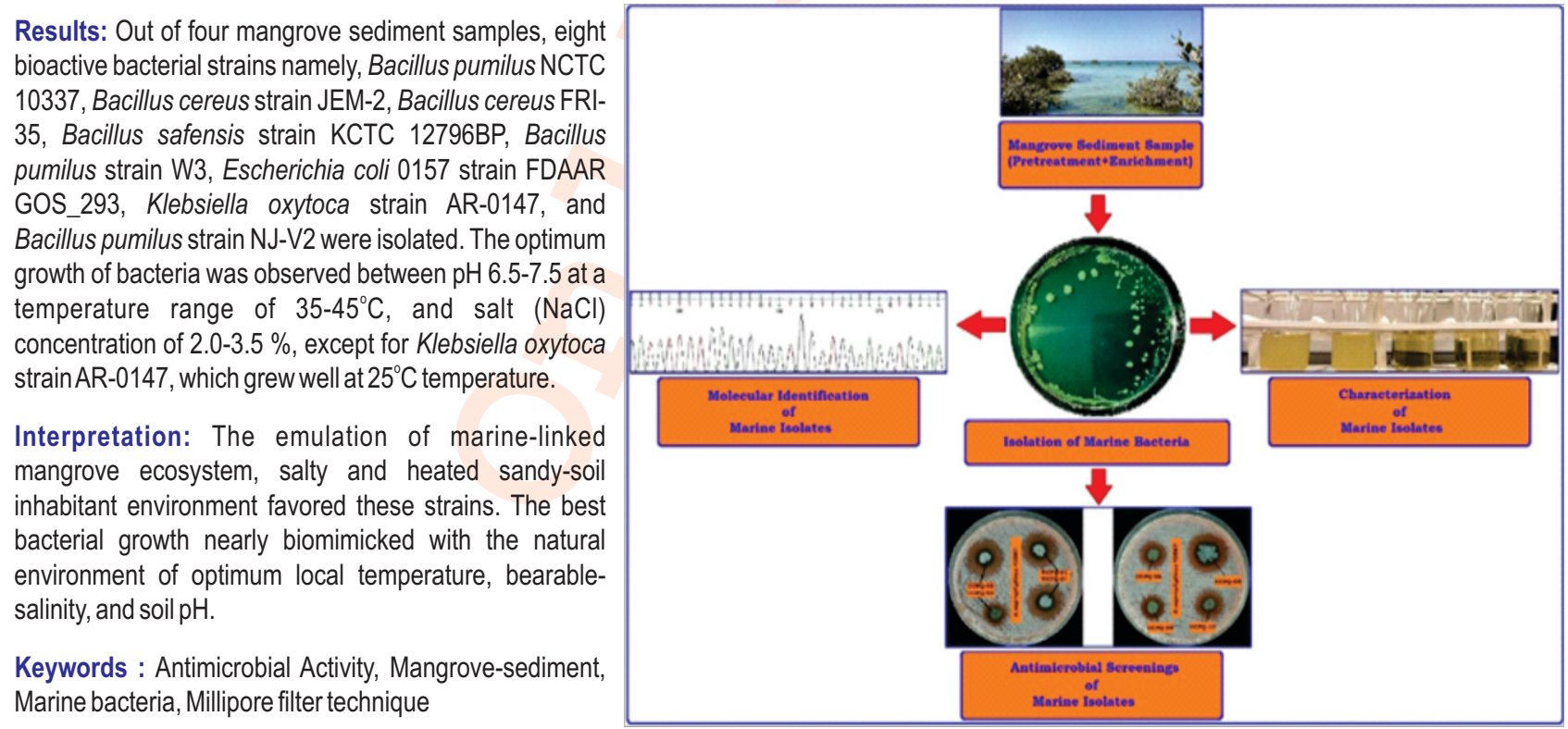

How to cite : Qureshi, K.A., M. Seroor, A. Al-Masabi, M.A. Saykhan, Y.A. Mutairi, G.O. Elhassan and R.A. Khan: Bio-characterizations of some marine bacterial strains isolated from mangrove sediment samples of four major cities of SaudiArabia. J. Environ. Biol., 41, 1003-1012 (2020). 


\section{Introduction}

The advent of new pathogenic microbes together with virulence of known microbes and developing resistance to known antibiotics together with severe pathological conditions associated with pathogenic bacterium infections are foremost challenges in the clinical settings dealing with mono and multidrug resistance infections. The health-care industry is in continuous demand for newer antibiotics through new drug discovery and lead generation to combat the spread, resistance, mutation, and virulence of the microbial entities. Discovery of new antimicrobial agents is pertinent to combat the approaching emergency in pathogen containment towards the treatment of infections caused by various multidrug-resistant organisms and newer virulence factors (Amin et al., 2016). One of the logical sources for new drug candidates and lead molecule discovery in antibiotic drug segment is in the search for microorganism strains capable of producing molecular entities possessing resistance to other microbes. The microorganisms fight the fellow microbes for survival and sustainability in the available environment for limited supply of space and nutrients. The microbes adept at various strategies to combat the competition for their survival, and the production of antimicrobial agents is among one of them. The lookout for new antimicrobial agents from microbes goes through the discovery and identification of new and rare strains of microorganisms producing endogenous antimicrobial agents which could also be obtained from known microorganisms including from the new habitat of microorganisms. Since antimicrobial agent's production by the microorganism is dependent on the challenges of the environment and the habitat, the soil is among one of the possible sources to search for new/modified strains and thereby finding possibly newer antimicrobial agents produced in response to the environmental challenges for the known and newer strains of the microorganisms. Soil rich in actinomycetes, eubacteria and fungi are important source for the antimicrobials (Donia et al., 2014), and in this perspective, the study was planned and executed.

The genus Streptomyces is one among the main genera of Actinomycetes while the other abundant genus, Bacillus, belongs to Eubacteria. These genera produce structurally-distinct bioactive compounds of antimicrobial nature (Naine et al., 2015). Bacillus is found in soil, water, and air with ranges of physiological characteristics allowing them to survive and sustain in moderate to extreme environments (Amin et al., 2015). The antimicrobial compounds produced by Bacillus strains are mostly proteinic (Violeta et al., 2011; Ghribi et al., 2012) and is widely used in healthcare and agricultural sectors for controlling various infectious diseases in humans, animals and plants (Leifert et al., 1995).

The coastal ecosystem of Saudi Arabia is largely unexplored and possibly a rich source of hereto unknown and unencountered strains of microorganisms which, in all probability, are expected to yield novel and efficient antimicrobial agents for future healthcare and other biomedical applications. In view of the above, the current study aimed to isolate, purify, and characterize the native antibiotic-producing bacterial strains present in the mangrove-sediments from four major coastal cities, namely, Jeddah, Yanbu, Dammam and Jubail of Saudi Arabia.

\section{Materials and Methods}

Sampling : The mangrove sediment samples were collected from the four major cities, i.e., Jeddah, Yambu, Dammam, and Jubail of Saudi Arabia by following a standard protocol (Alanazi et al., 2016).

Test organisms and test medium : Staphy ATCC 25923, Staphylococcus aureus ATCC 29213, Methicillinresistant Staphylococcus aureus-A, Methicillin-resistant Staphylococcus aureus-B, Staphylococcus epidermidis ATCC 12228, Staphylococcus saprophyticus ATCC 43867, Bacillus cereus ATCC 10876, Streptococcus pyogenes (A) ATCC 27736, Streptococcus pneumoniae ATCC 49619, Enterococcus faecalis ATCC 29212, Escherichia coli ATCC 10536, Escherichia coli ATCC 25922, Klebsiella pneumoniae ATCC 27736 , Pseudomonas aerugenosa ATCC 9027, Proteus mirabilis ATCC 29906, Proteus vulgaris ATCC 6380, Salmonella typhimurium ATCC 13311, Shigella flexneri ATCC 12022, Candida albicans ATCC 10231 and Aspergillus niger ATCC 6275 were used as test organisms.

International Streptomyces Project Media (ISP-4), modified tryptic soy broth [1\% starch; 50:50 (seawater: distilled water)], chocolate tryptic soy agar, chocolate Mueller-Hinton agar were used as test media. The test media were purchased from HiMedia, Mumbai, India.

Pretreatment and enrichment : To isolate native marine bacteria, the mangrove-sediment samples were air-dried for a week at room temperature $\left(\mathrm{RT}, 25 \pm 2^{\circ} \mathrm{C}\right)$, and after a week of incubation, the samples were placed in a hot-air oven at $60^{\circ} \mathrm{C}$ for 1 $\mathrm{hr}$ to prevent contamination by other microbial flora. The selective enrichment technique and two enrichment media, i.e., ISP4 and modified tryptic soy broth were used for isolating of marine bacteria. Ten gram of each mangrove-sediment sample was mixed separately with $100 \mathrm{ml}$ of ISP4, and modified tryptic soy broth.

The mixtures were incubated at RT $\left(25 \pm 2^{\circ} \mathrm{C}\right)$ at $150 \mathrm{rpm}$ in a shaker incubator (Benchtop Shaking Incubator, LBSIO-202, Labocon Scientific Limited, UK) for 7 days according to Hirsch and Christensen, 1983; Baskaran et al., 2011.

Isolation of marine bacteria : Following the completion of incubation, serial dilutions $\left(10^{-1}\right.$ to $\left.10^{-5}\right)$ of each enriched sample were prepared in sterile phosphate buffer saline $(\mathrm{pH} 7.0)$ (Bhate, 1955; Alanazi et al., 2016). The selective Millipore® filter 
technique was followed for isolating marine bacteria (Hirsch and Christensen, 1983) wherein sterile Millipore $₫$ filter paper $(0.45$ $\mu \mathrm{M}$ pore size, $100 \mathrm{~mm}$ diameter) was placed on a modified tryptic soy-agar plate, and $100 \mu$ of enriched sample ( $10^{-4}$ dilution) was inoculated and spread-out. The inoculated plates were incubated at RT $\left(25 \pm 2^{\circ} \mathrm{C}\right)$ for 7 days, and then filter paper was removed with the help of a sterile forceps and re-incubated at RT $\left(25 \pm 2^{\circ} \mathrm{C}\right)$ for 10 days. Colonial characteristics of isolated organisms were recorded and sub-culturing was performed on sterile tryptic soyagar plates.

Antimicrobial screening : Antimicrobial screenings of isolated bacteria were performed performed by following the spot inoculation method as described by Alanazi et al. (2016). Sterile chocolate Mueller-Hinton agar plates for antibacterial screening and sterile chocolate tryptic soy-agar plates for antifungal screening were used as test media. A $100 \mu$ l suspension of each test organism ( 0.5 MacFarland) was inoculated and spread out separately on the test medium plates, and a loop full inoculum of each isolate was spotted on the surface of individual test media plate. After inoculation, all the plates were incubated at $4{ }^{\circ} \mathrm{C}$ for 30 min, followed by incubation of chocolate Mueller-Hinton agar plates at $35 \pm 2{ }^{\circ} \mathrm{C}$ for $24-48 \mathrm{hrs}$ and the chocolate tryptic soy-agar plates at $25 \pm 2{ }^{\circ} \mathrm{C}$ for $48-120 \mathrm{hrs}$. The results of antimicrobial screenings were recorded by observing the diameter of zone of inhibition against test organisms.

Identification of bioactive marine bacteria : Bioactive bacteria were selected based on the zone of inhibition against test organisms. Phenotypic characterizations of bioactive strains were carried out by observing the morphological and colony characteristics. Genotypic characterization was carried out by next-generation 16S rRNA sequencing method as described by Alanazi et al. (2016).

Characterization of bioactive marine bacteria : Bioactive marine isolates were characterized by following the method of Qureshi et al. (2016). The effects of $\mathrm{pH}$, temperature, and salt $(\mathrm{NaCl})$ concentrations on the growth of bacteria were investigated. Tryptic soy-broth was used as test medium.

Determination of $\mathrm{pH}$ : The effect of $\mathrm{pH}$ on the growth of isolates was determined by observing the optimum growth at different $\mathrm{pH}$ conditions, i.e., 4.0, 4.5, 5.0, 5.5, 6.0, 6.5, 7.0, 7.5, and 8.0 through changing the $\mathrm{pH}$ of the growth media. Both $1 \mathrm{~N} \mathrm{HCl}$ and $1 \mathrm{~N} \mathrm{KOH}$ aqueous solutions were used to adjust the required $\mathrm{pH}$. Three replicates of each treatment were performed. After the inoculation of seed-cultures, the initial optical density of the growth was determined at ${ }^{\wedge} 600 \mathrm{~nm}$ wavelength of the spectrophotometer. The deionized water was used as blank. All the treatments were incubated at $35 \pm 2^{\circ} \mathrm{C}$ for $48 \mathrm{hrs}$ and postincubation, the optical densities of the treatments were observed at ${ }^{\wedge} 600 \mathrm{~nm}$ wavelength. The differences in the optical densities (zero and $48 \mathrm{hrs}$ incubations) were recorded wherein the net increase in the optical density indicated the positive-growth of the bacteria.

Effect of temperature : The effect of temperature on the growth of the bioactive bacteria was determined by incubating the isolated bacterial strains at different temperatures, i.e., 25, 30, 35, 40,45 , and $50^{\circ} \mathrm{C}$. The three replicates of each treatment were performed. After the inoculation of seed-cultures in the growth media at optimum $\mathrm{pH}$, the initial optical density of the growths was determined at ${ }^{\wedge} 600 \mathrm{~nm}$ wavelength of the spectrophotometer. The deionized water was used as blank. All the treatments were incubated at different temperatures, i.e., 25, 30, 35, 40, 45, and 50 ${ }^{\circ} \mathrm{C}$ for $48 \mathrm{hrs}$ and post-incubation, the optical densities of the treatments were observed at ${ }^{\wedge} 600 \mathrm{~nm}$ wavelength. The differences in the optical densities (zero $\mathrm{hr}$ and $48 \mathrm{hrs}$ of incubations) were recorded wherein the net increase in the optical density indicated the positive-growth of the bacteria.

Effect of $\mathrm{NaCl}$ concentrations : The effect of salt concentrations on the growth of bioactive bacteria was determined by incubating isolated bacteria at different $\mathrm{NaCl}$ concentrations, i.e., 1.0, 1.5, 2.0, 2.5, 3.0, 3.5, 4.0, 4.5, 5.0, and $6.0 \%$. Three replicas of each treatment were performed. After inoculation of seed-cultures in the growth media at optimum $\mathrm{pH}$, the initial optical density of the growth was determined at ${ }^{\wedge} 600 \mathrm{~nm}$ wavelength of the spectrophotometer. Deionized water was used as blank. All the treatments were incubated at an optimum temperature for $48 \mathrm{hrs}$ and post-incubation, the optical densities of treatments were observed at ${ }^{\wedge} 600 \mathrm{~mm}$. Differences in the optical densities (zero hr and $48 \mathrm{hrs}$ of incubations) were recorded wherein the net increase in the optical density indicated the positive-growth of bacteria.

Statistical analyses: The difference in the results of mean antimicrobial scores of marine isolates were analyzed by following the ANOVA test using SPSS version 20.0 software (IBM, USA). No significant difference at $p=0.05$ level was considered.

\section{Results and Discussion}

Out of four mangrove sediment samples, ten different types of bacterial strains were isolated. Temporary identification codes; UCPQ-01 to UCPQ-10 were assigned to all the isolated bacterial strains. Isolated colonies were purified for further investigation and kept at $2-4^{\circ} \mathrm{C}$ until further use.

Out of ten isolates, only eight bacterial strains namely, Bacillus pumilus NCTC 10337 (UCPQ-01), Bacillus cereus strain JEM-2 (UCPQ-02), and Klebsiella oxytoca strain AR-0147 (UCPQ-08) were isolated from Jeddah city's mangrove sediment sample whereas Bacillus cereus FRI-35 (UCPQ-03), Bacillus safensis strain KCTC 12796BP (UCPQ-04) were isolated from Dammam city's mangrove sediment sample, and Escherichia coli 0157 strain FDAAR GOS_293 (UCPQ-06) and Bacillus pumilus strain NJ-V2 (UCPQ-10) were isolated from Jubail city's 
mangrove sediment sample, and Bacillus pumilus strain W3 (UCPQ-05) was isolated from the Yanbu city's mangrove sediment sample, exhibited significant antibacterial activity against test organisms. The results are summarized in Table 1.

Out of ten bacterial isolates, only five isolates namely as Bacillus cereus strain JEM-2 (UCPQ-02), Bacillus cereus FRI-35 (UCPQ-03), Bacillus pumilus strain W3 (UCPQ-05), Escherichia coli 0157 strain FDAAR GOS_293 (UCPQ-06), and Klebsiella oxytoca strain AR-0147 (UCPQ-08) exhibited antifungal activity against Candida albicans ATCC 10231 only. The results are summarized in Table 2. The results of antibacterial activity suggested that out of ten isolated bacterial strains only eight bacterial strains showed antibacterial activity against Grampositive bacteria whereas some of the isolated strains, i.e., Bacillus pumilus NCTC 10337 (UCPQ-01), Bacillus cereus strain JEM-2 (UCPQ-02), Bacillus safensis strain KCTC 12796BP (UCPQ-04), Bacillus pumilus strain W3 (UCPQ-05), Escherichia coli 0157 strain FDAAR GOS_293 (UCPQ-06), and Klebsiella oxytoca strain AR-0147 (UCPQ-08) exhibited antibacterial activity against Escherichia coliATCC 10536.

The results of antifungal activity suggested that out of eight selected bacterial strains only five showed antifungal

Table 1 : Antibacterial screening of isolated bacterial strains*

\begin{tabular}{|c|c|c|c|c|c|c|c|c|c|c|c|c|c|c|c|c|c|c|c|c|}
\hline \multirow[t]{2}{*}{ Code } & \multirow[t]{2}{*}{ Isolate ID } & \multicolumn{19}{|c|}{ Test organisms } \\
\hline & & A & B & C & D & $E$ & $\mathbf{F}$ & G & $\mathrm{H}$ & I & $\mathrm{J}$ & $\mathrm{K}$ & $\mathrm{L}$ & $M$ & $\mathrm{~N}$ & 0 & $\mathbf{P}$ & $Q$ & $\mathbf{R}$ & $x$ \\
\hline UCPQ-1 & $\begin{array}{l}\text { Bacillus pumilus } \\
\text { NCTC } 10337\end{array}$ & 1 & 1 & 1 & 1 & 1 & 1 & 0 & 1 & 0 & 0 & 1 & 0 & 0 & 0 & 0 & 0 & 0 & 0 & 8 \\
\hline UCPQ-2 & $\begin{array}{l}\text { Bacillus cereus } \\
\text { strain JEM-2 }\end{array}$ & 1 & 1 & 1 & 1 & 1 & 1 & 0 & 1 & 0 & 0 & 1 & 0 & 0 & 0 & 0 & 0 & 0 & 0 & 8 \\
\hline UCPQ-3 & $\begin{array}{l}\text { Bacillus cereus } \\
\text { FRI- } 35\end{array}$ & 0 & 0 & 1 & 1 & 1 & 1 & 0 & 1 & 0 & 0 & 0 & 0 & 0 & 0 & 0 & 0 & 0 & 0 & 5 \\
\hline UCPQ-4 & $\begin{array}{l}\text { Bacillus safensis } \\
\text { strain KCTC 12796BP }\end{array}$ & 1 & 1 & 1 & 1 & 1 & 1 & 0 & 0 & 0 & 0 & 1 & 0 & 0 & 0 & 0 & 0 & 0 & 0 & 7 \\
\hline UCPQ-5 & $\begin{array}{l}\text { Bacillus pumilus } \\
\text { strain W3 }\end{array}$ & 1 & 1 & 1 & 1 & 1 & 1 & 0 & 1 & 0 & 0 & 1 & 0 & 0 & 0 & 0 & 0 & 0 & 0 & 8 \\
\hline UCPQ-6 & $\begin{array}{l}\text { Escherichia coli0157 } \\
\text { strain FDAAR G0S_293 }\end{array}$ & 1 & 1 & 1 & 1 & 1 & 1 & 0 & 1 & 0 & 0 & 1 & 0 & 0 & 0 & 0 & 0 & 0 & 0 & 8 \\
\hline UCPQ-8 & $\begin{array}{l}\text { Klebsiella oxytoca } \\
\text { strain AR-0147 }\end{array}$ & 1 & 1 & 1 & 1 & 1 & 1 & 0 & 1 & 0 & 0 & 1 & 0 & 0 & 0 & 0 & 0 & 0 & 0 & 8 \\
\hline UCPQ-10 & $\begin{array}{l}\text { Bacillus pumilus } \\
\text { strain NJ-V2 }\end{array}$ & 1 & 1 & 1 & 1 & 1 & 1 & 0 & 1 & 0 & 0 & 0 & 0 & 0 & 0 & 0 & 0 & 0 & 0 & 7 \\
\hline
\end{tabular}

${ }^{*} 1=$ antibacterial activity present, $0=$ antibacterial activity absent, $X=$ total antibacterial score, $A=$ Staphylococcus aureus ATCC 25923, B=Staphylococcus aureus ATCC 29213, C=Methicillin-resistant Staphylococcus aureus-A, D=Methicillin-resistant Staphylococcus aureus-B, $\mathrm{E}=$ Staphylococcus epidermidis ATCC 12228, $\mathrm{F}=$ Staphylococcus saprophyticus ATCC 43867, G=Bacillus cereus ATCC 10876, $\mathrm{H}=$ Streptococcus pyogenes (A) ATCC 27736, I=Streptococcus pneumoniae ATCC 49619, J=Enterococcus faecalis ATCC 29212, $\mathrm{K}=$ Escherichia coli ATCC 10536, L=Escherichia coli ATCC 25922, M=Klebsiella pneumoniae ATCC 27736, N=Pseudomonas

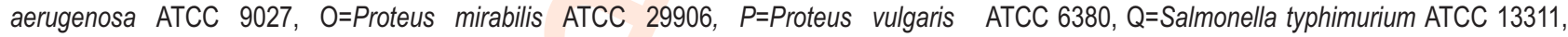
$\mathrm{R}=$ Shigella flexneri ATCC 12022

Table 2 : Antifungal screening of isolated bacterial strains ${ }^{* \star}$

\begin{tabular}{lllll}
\hline Code & Isolate ID & \multicolumn{2}{l}{ Test organisms } & \\
\cline { 3 - 5 } & & $\begin{array}{l}\text { Candida albicans } \\
\text { ATCC 10231 }\end{array}$ & $\begin{array}{l}\text { Aspergillus niger } \\
\text { ATCC 6275 }\end{array}$ & $\begin{array}{l}\text { Total Anti-fungal } \\
\text { Score }\end{array}$ \\
\hline UCPQ-2 & Bacillus cereus strain JEM-2 & 1 & 0 & 1 \\
UCPQ-3 & Bacillus cereusFRI-35 & 1 & 0 & 1 \\
UCPQ-5 & Bacillus pumilus strain W3 & 1 & 0 & 1 \\
UCPQ-6 & Escherichia coli0157 strain FDAARGOS_293 & 1 & 0 & 1 \\
UCPQ-8 & Klebsiella oxytocastrainAR-0147 & 1 & 0 & 1 \\
\hline
\end{tabular}

\footnotetext{
**1=antifungal activity present; $0=$ antifungal activity absent; $X=$ total antifungal score
} 
activity against Candida albicans ATCC 10231. The results of antifungal activity were not encouraging. Based on the observations, the eight isolates were selected for further investigations. The results of phenotypic characterization are summarized in Table 3. The statistical analyses confirmed that there was no significant difference $(P>0.05)$ in the antimicrobial activities of marine isolates.

The results of next-generation 16S rRNA sequencing analysis were BLASTED on the website of NCBI (Gene data bank) and the isolated bacterial strains were identified as Bacillus pumilus NCTC 10337 (UCPQ-01), Bacillus cereus strain JEM-2 (UCPQ-02), Bacillus cereus FRI-35 (UCPQ-03), Bacillus safensis strain KCTC 12796BP (UCPQ-04), Bacillus pumilus strain W3 (UCPQ-05), Escherichia coli 0157 strain FDAAR GOS 293 (UCPQ-06), and Klebsiella oxytoca strain AR-0147 (UCPQ08), and Bacillus pumilus strain NJ-V2 (UCPQ-10). The phylogenetic trees were constructed with neighbor-joining method, and the results are illustrated in Fig. 1 (a-h).

The ongoing results suggested that the predominant bioactive bacterial genus found in the mangrove sediment samples was Bacillus when compared among all the four locations of Saudi Arabia. Escherichia coli 0157 strain FDAAR GOS_293 and Klebsiella oxytoca strain AR-0147 were also present in the mangrove sediment samples. The results further illustrated that no actinomycetes strain was isolated from any of the four samples collected from four locations.

The results of characterizations of isolated strains showed that the optimum growth of bioactive bacteria were observed at a $\mathrm{pH}$ range of $6.5-7.5$, temperature range of $25-45^{\circ} \mathrm{C}$, and 2.0-3.5 \% of $\mathrm{NaCl}$ concentrations. The results are summarized in Fig. 2 (a-c).

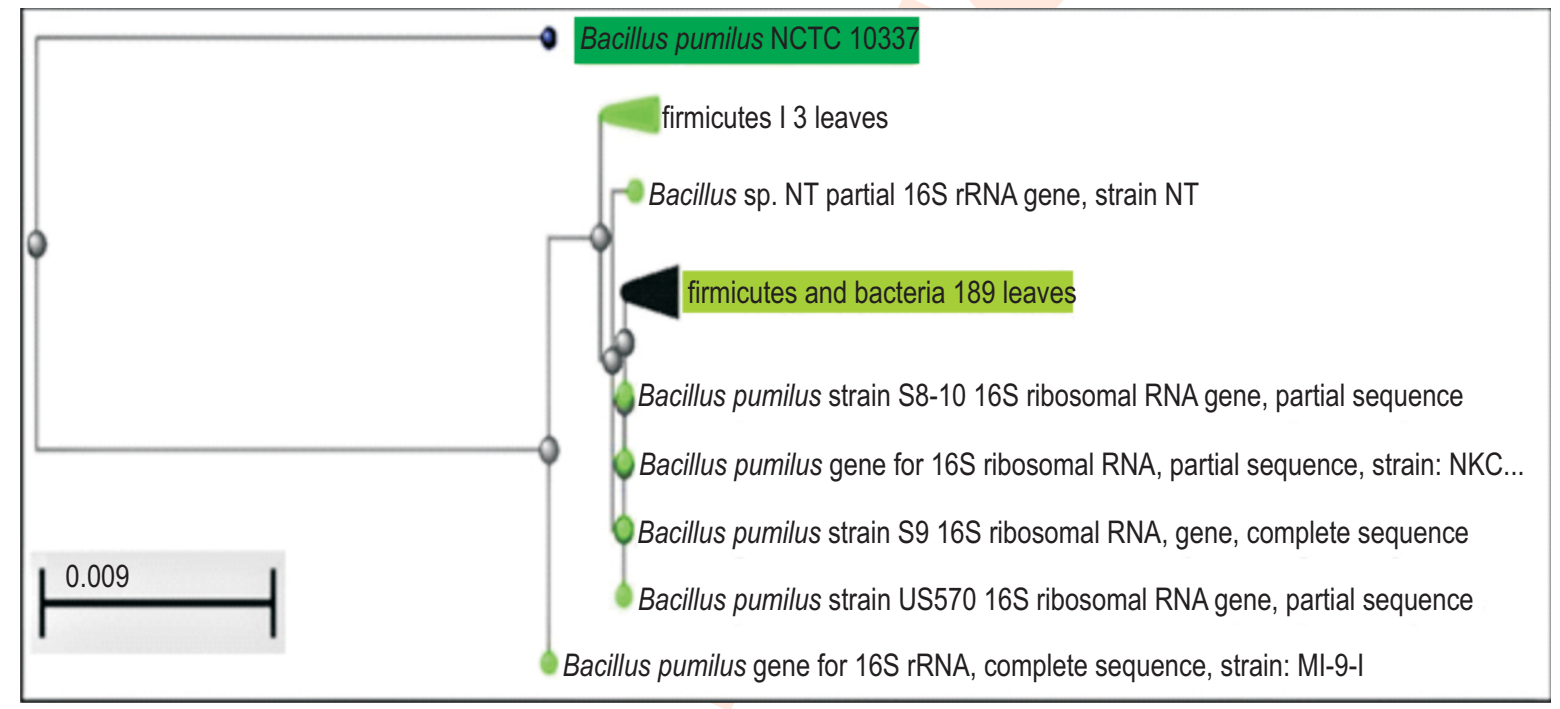

Fig. 1 (a) : Bacillus pumilus NCTC 10337.

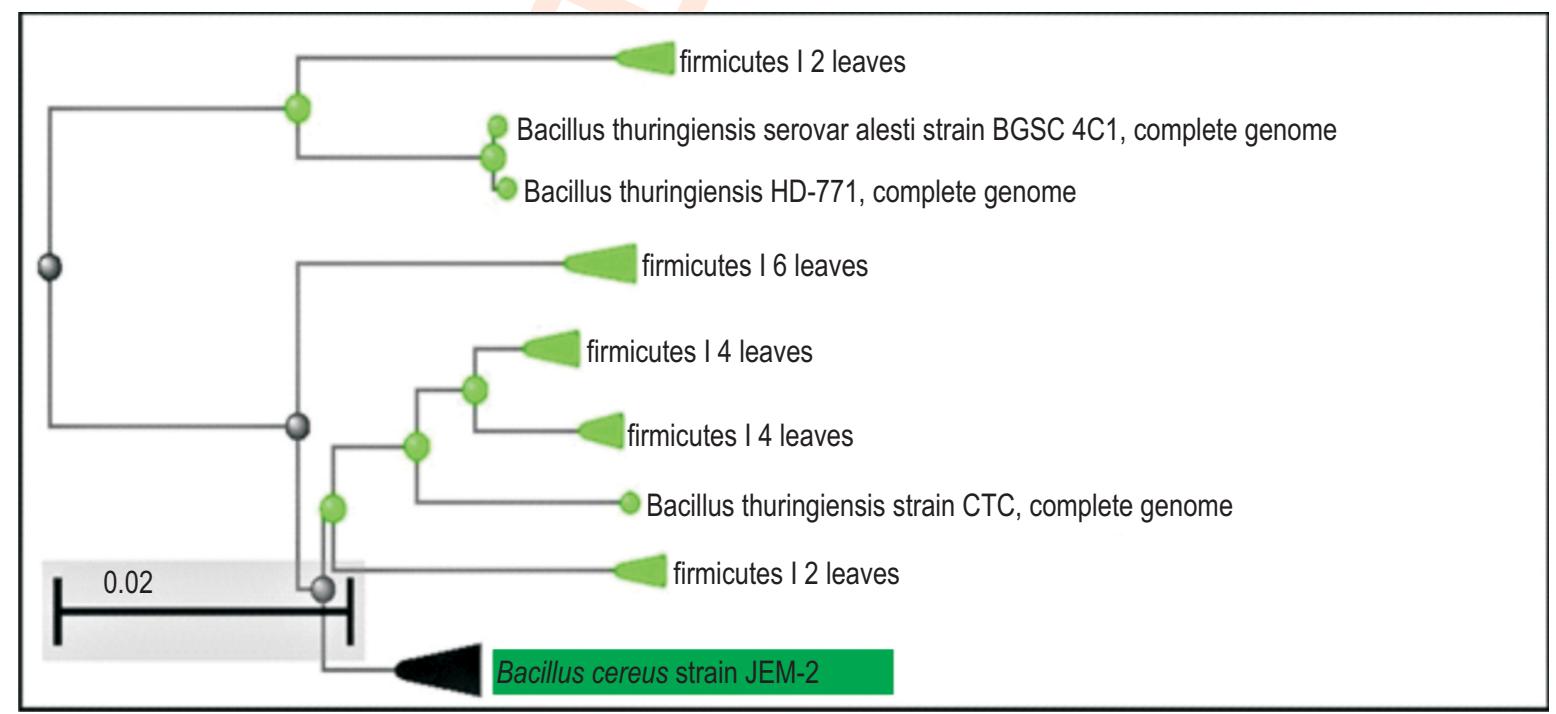

Fig. 1 (b) : Bacillus cereus strain JEM-2. 


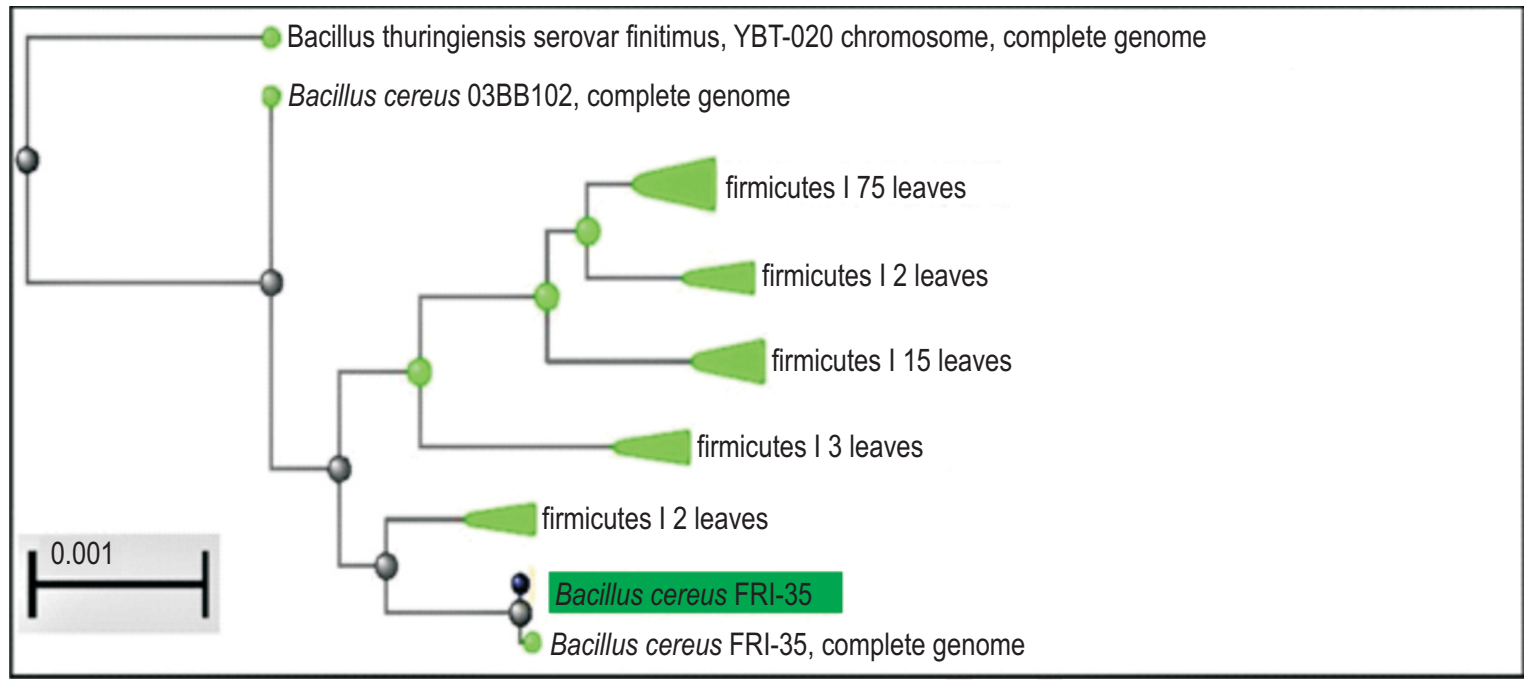

Fig. 1 (c) : Bacillus cereus FRI-35

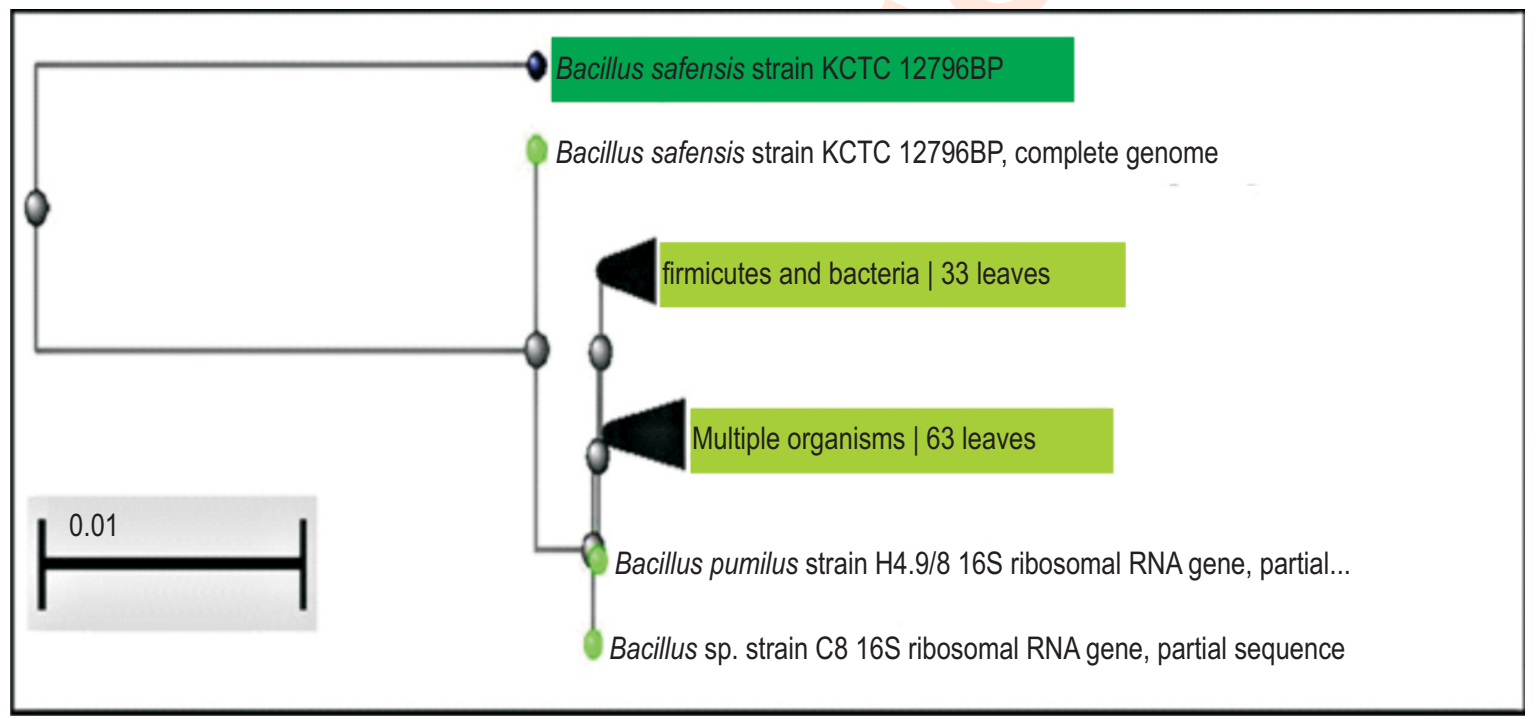

Fig. 1 (d) : Bacillus safensis strain KCTC 12796BP.

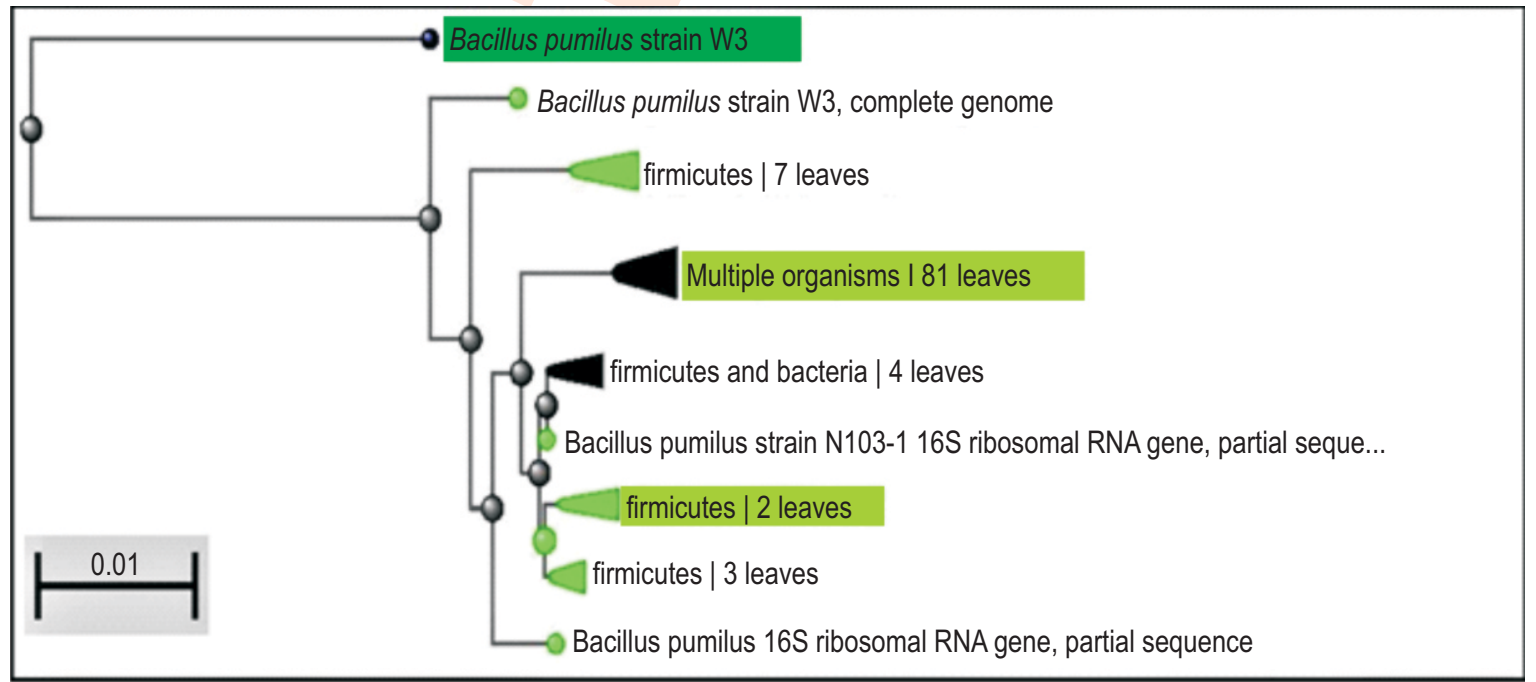

Fig. 1 (e) : Bacillus pumilus strain W3. 


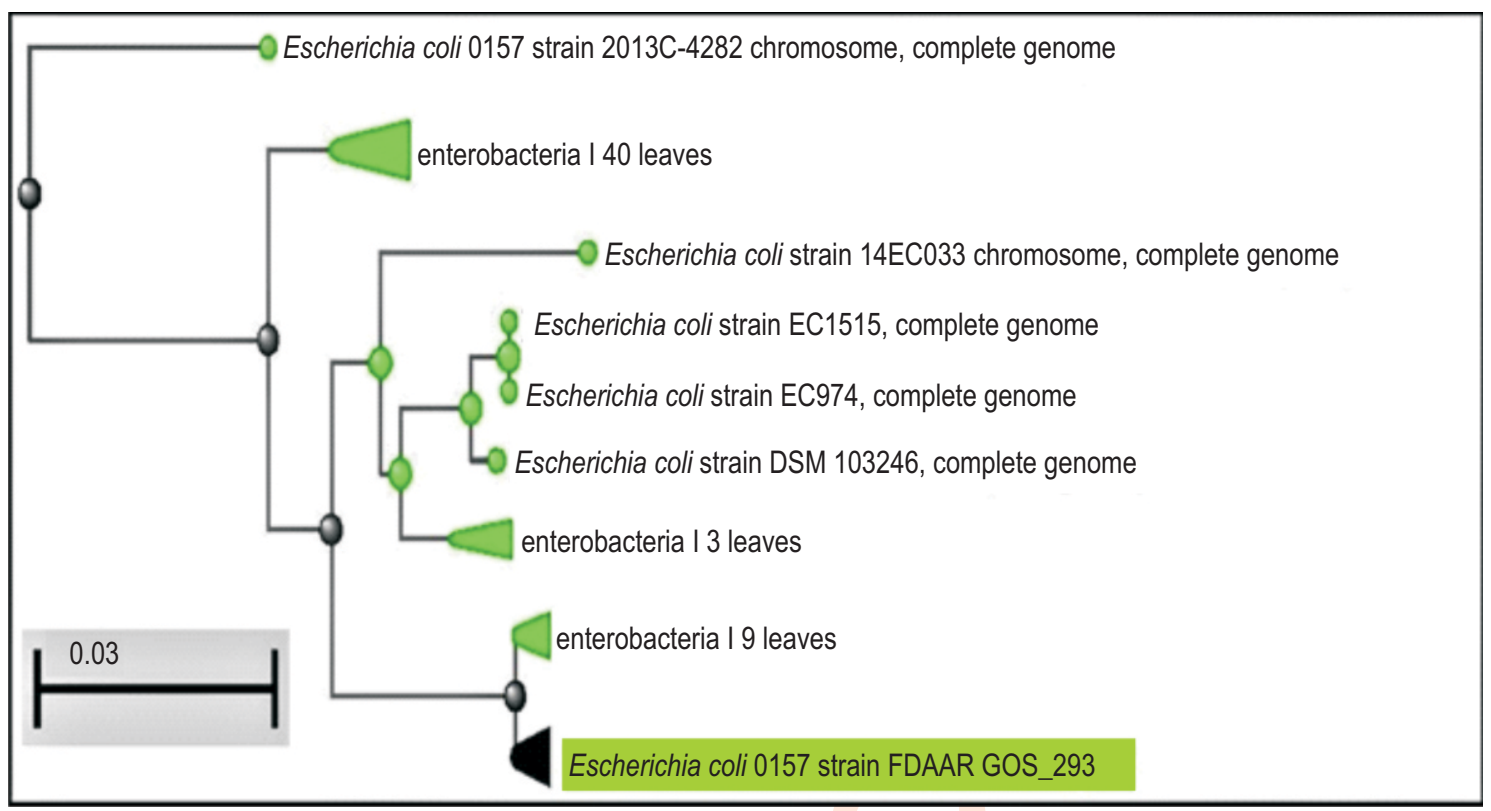

Fig. 1 (f) : Escherichia coli 0157 strain FDAAR GOS_293.

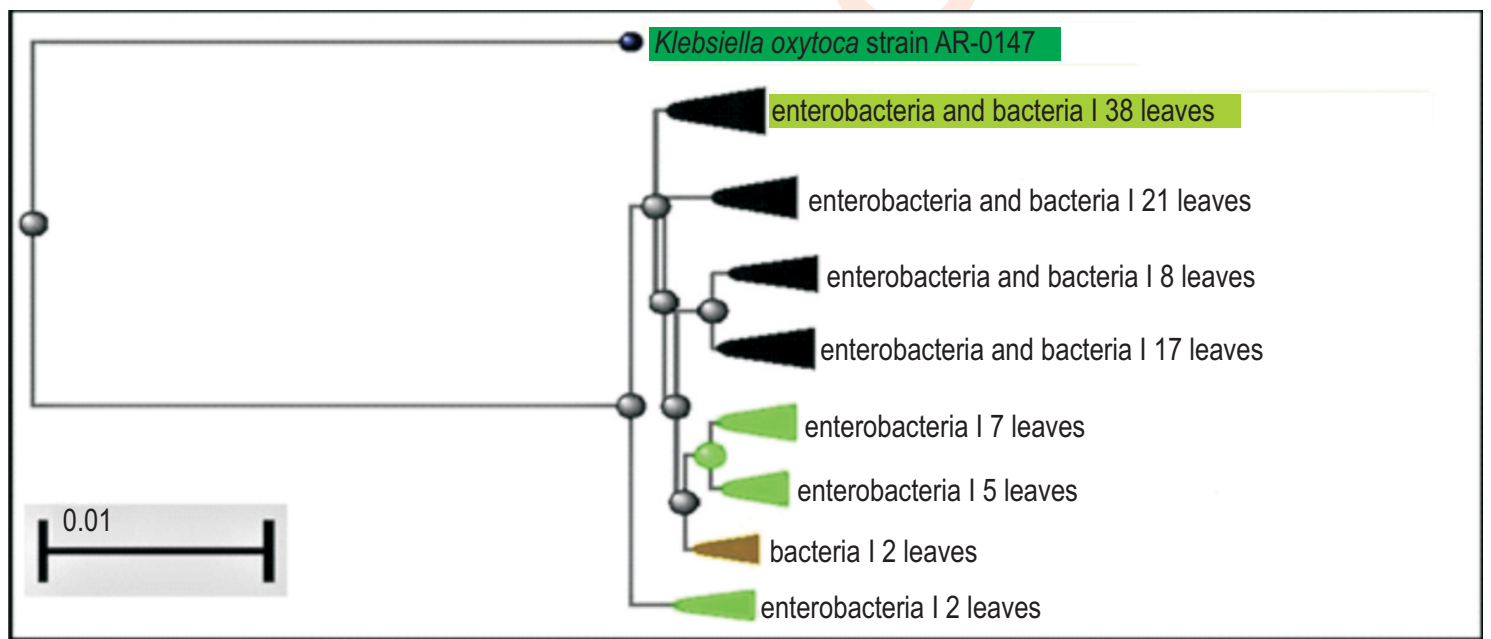

Fig. 1 (g) : Klebsiella oxytoca strain AR-0147.

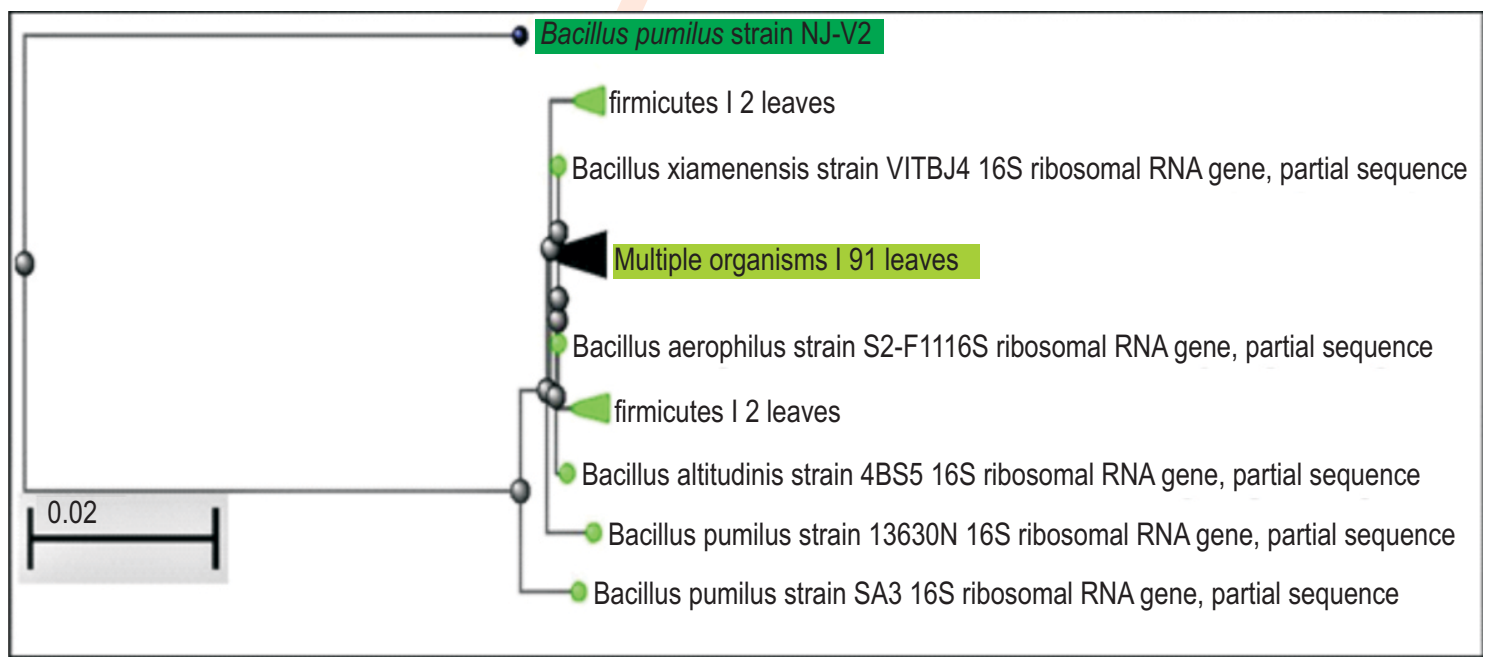

Fig. 1 (h) : Bacillus pumilus strain NJ-V2. 

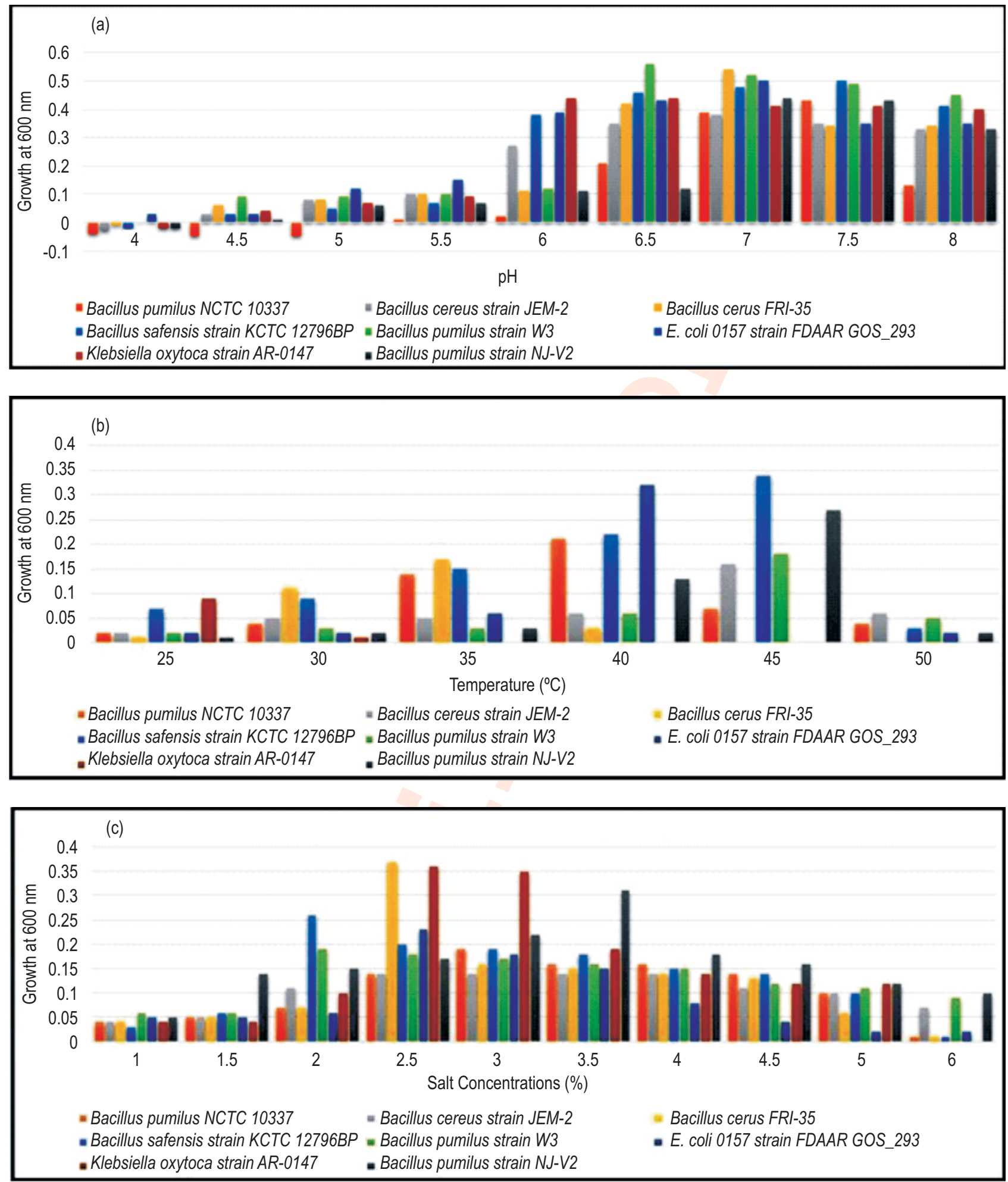

Fig. 2 (a-c) : Effect of pH, temperature and salt concentration on the growth of bioactive marine bacteria.

The result of characterization suggested that majority of bacteria were mild to moderate halophilic, and grew well in the presence of 2.0-3.5 \% NaCl concentrations in media with $\mathrm{pH}$ ranging between $6.5-7.5$ and at temperature between $35-45^{\circ} \mathrm{C}$.
Some of the previous reports support our study. Bhate (1955) found that Bacillus pumilus produces a substance with antibacterial activity and it was identified as pumilin, while another study showed that Bacillus pumilus contained antimicrobial 
Table 3: Phenotypic characteristics of isolated bacterial strains

\begin{tabular}{lllllllll}
\hline Characteristics & \multicolumn{7}{c}{ Isolates } \\
\cline { 2 - 8 } & UCPQ-1 & UCPQ-2 & UCPQ-3 & UCPQ-4 & UCPQ-5 & UCPQ-6 & UCPQ-8 & UCPQ-10 \\
\hline Shape & Circular & Circular & Circular & Circular & Circular & Circular & Circular & Circular \\
Size & $2-3 \mathrm{~mm}$ & $2-3 \mathrm{~mm}$ & $2-3 \mathrm{~mm}$ & $2-3 \mathrm{~mm}$ & $2-3 \mathrm{~mm}$ & $2-3 \mathrm{~mm}$ & $2-3 \mathrm{~mm}$ & $2-3 \mathrm{~mm}$ \\
Elevation & Raised & Raised & Raised & Raised & Raised & Low convex & Dome-shaped & Raised \\
Margin & Undulate & Undulate & Undulate & Undulate & Entire & Entire & Entire & Entire \\
Opacity & Opaque & Opaque & Opaque & Opaque & Opaque & Opaque & Opaque & Opaque \\
Consistency & Viscid & Viscid & Viscid & Viscid & Viscid & Butyrous & Mucoid & Viscid \\
Emulsifiability & Difficult-to- & Difficult-to- & Difficult-to- & Difficult-to- & Difficult-to- & Emulsifiable & Emulsifiable & Difficult-to- \\
& emulsify & emulsify & emulsify & emulsify & emulsify & & Emulsify \\
Odor & Earthy & Earthy & Earthy & Earthy & Earthy & Fecal odor & Fecal odor & Earthy \\
Gram reaction & Gram-positive & Gram-positive & Gram-positive & Gram-positive & Gram-positive & Gram-negative & Gram-negative & Gram-positive \\
\hline
\end{tabular}

activity, but the bioactive compound was not isolated from the strain (Gilliver, 1949). In an another attempt Dvonch and Benedict (1953) succeeded in isolating the antimicrobial agent from another Bacillus pumilus strain which was structurally similar to antibiotic subtenolin. Borowski et al. (1953) isolated another antibiotic tetaine from Bacillus pumilus. The study by Singh et al. demonstrated that Bacillus pumilus (NKCM 8905), and Bacillus pumilus (AB211228) produce antimicrobial agents (Singh et al., 2016) Wessel and Flugge researchers described the $B$. pumilus CL45 strain's antibiotic as antagonistic to plant pathogens but lacked the full antimicrobial profile (Wessel and Flugge, 1984; Valgas et al., 2007). However, some recent studies related to Bacillus pumilus NJ-M2 and Bacillus pumilus SAFR-032 strains to produce antimicrobial compounds against some of the selected test pathogenic organisms, however, the isolated pure sample and identified antimicrobial compound(s) from these two Bacillus strains were not further investigated (Alanazi et al., 2016; Qureshi et al., 2016). In another study, Klebsiella oxytoca strain Aft-7 was found to be detoxified molybdenum and degrade detergents. The observation categorizes this bacterium as an important tool for bioremediation purpose (Masdor et al., 2015).

Reports on Escherichia coli 0157 strain FDAAR GOS_293 and Klebsiella oxytoca strain AR-0147 with reference to the production of antimicrobial compound(s) against Staphylococcus aureus ATCC 25923, Staphylococcus aureus ATCC 29213, Methicillin-resistant Staphylococcus aureus-A, Methicillin-resistant Staphylococcus aureus-B, Staphylococcus epidermidis ATCC 12228, Staphylococcus saprophyticus ATCC 43867, Streptococcus pyogenes (A) ATCC 27736, Escherichia coli ATCC 10536, and Candida albicans ATCC 10231 are meager. The current findings are the first report in this domain, that opens up the field for further investigations to isolate, purify, and characterize the chemical structure of antimicrobial agent(s) produced by these strains obtained from the coastal mangrove sediment soils. The study identifies multiple sources of antimicrobial agents for further investigation, in addition to providing the antimicrobial profile of these bioactive bacterial strains, isolated from different soil samples. The first step in procuring, and identifying the soil sources for environmentallychallenged bacterial strains, the antimicrobial activity of these isolated bacteria, and the antimicrobial profiles of majority of isolates, and purified bacteria have been achieved to pave the way for the isolation, purification, structure elucidation and antimicrobial activity confirmation of probable new/modified molecular entities for the small and large-molecule drug discovery, or known antibiotics activity re-evaluation of new and virulent strains which will add to another confirmed source for the bacterium for in time of need utilization.

In conclusion, the ongoing results suggest that the eight fully identified, marine bioactive bacterial strains can be used for the production of new antimicrobial agents which is deeply desired. It may also lead towards the discovery of new molecular templates, and compounds for discovery of drugs in the antimicrobial segment. Thus, the above results warrant further investigations to stabilize the full antimicrobial profiles of these marine isolates.

\section{Acknowledgment}

Authors thank Dean, Unaizah College of Pharmacy (Qassim University), Unaizah, Saudi Arabia for providing technical help and financial resources during this study.

\section{References}

Alanazi, A.S., K.A. Qureshi, G.O. Elhassan and E.I. El-Agamy: Isolation, purification, and characterization of antimicrobial agent antagonistic to Escherichia coli ATCC 10536 produced by Bacillus pumilus SAFR-032 isolated from the soil of Unaizah, Al Qassim province of Saudi Arabia. Pak. J. Biol. Sci., 19, 191-201 (2016).

Amin, M., Z. Rakhisi and A.Z. Ahmady: Isolation and identification of Bacillus species from soil and evaluation of their antibacterial properties. Avicenna J. Clin. Microb. Infect. 2, e23233 (2015).

Baskaran, R., R. Vijayakumar and P.M. Mohan: Enrichment method for the isolation of bioactive actinomycetes from mangrove sediments of Andaman Islands, India. Malay. J. Microbiol., 7, 22-28 (2011). 
Bhate, D.S. and A. Pumilin: New antibiotic from Bacillus pumilus. Nature, 175, 816-817 (1955).

Borowski, E.: Isolation of pure tetaine-an antibiotic produced by the theta strain of Bacillus pumilus. Przemysl. Chem., 9, 503 (1953).

Donia, M.S., P. Cimermancic, C.J. Schulze, L.C.W. Brown, J. Martin, M. Mitreva, J. Clardy, R.G. Linington and M.A. Fischbach: A systematic analysis of biosynthetic gene clusters in the human microbiome reveals a common family of antibiotics. Cell, 158, 1402(2014).

Dvonch, W. and R.G. Benedict: Elaboration of a subtenolin-like antibiotic by Bacillus pumilus. Antibiotics Chemother., 3, 192-194 (1953).

Ghribi, D., L. Abdelkefi-Mesrati, I. Mnif, R. Kammoun, I. Ayadi, I. Saadaoui, S. Maktouf and S. Chaabouni-Ellouze: Investigation of antimicrobial activity and statistical optimization of Bacillus subtilis SPB1 biosurfactant production in solid-state fermentation. J. Biomed. Biotechnol., 2012, 373682 (2012).

Gilliver, K.: The antibacterial properties of some species of aërobic spore-forming bacilli. Br.J.Exp. Pathol., 30, 214-220 (1949).

Hasan, F., S. Khan, A.A. Shah and A. Hameed: Production of antibacterial compounds by free and immobilized Bacillus pumilus SAF1. Pak. J. Bot., 41, 1499-1510 (2009).

Hirsch, C.F. and D.L. Christensen: A novel method for selective isolation of actinomycetes. App. Env. Microbiol., 46, 925-929 (1983).

Leifert, C., H. Li, S. Chidburee, S. Hampson, S. Workman, D. Sigee, H.A. Epton and A. Harbour: Antibiotic production and biocontrol activity by Bacillus subtilis CL27 and Bacillus pumilus CL45. J. Appl. Bacteriol., 78, 97-108(1995).
Masdor, N., M.S.A. Shukor, A. Khan, M.I.E.B. Halmi, S.R.S. Abdullah, N.A. Shamaan and M.Y. Shukor: Isolation and characterization of a molybdenum-reducing and SDS degrading Klebsiella oxytoca strain Aft-7 and its bioremediation application in the environment. Biodiversitas, 16, 238-246 (2015).

Naine, S.J., C.S Devi, V. Mohanasrinivasan and B. Vaishnavi: Antimicrobial, antioxidant and cytotoxic activity of marine Streptomyces parvulus VITJS11 crude extract. Braz. Arch. Bio. Tech., 58, 198-207 (2015).

Qureshi, K.A., A.D. Bholay, G.O. Elhassan, R.A. Khan and E.I. ElAgamy: Isolation, purification, and characterization of bacteriocin produced by Bacillus pumilus NJ-M2; A future biopreservative. Int. J. Biol. Pharm. Allied. Sci., 5, 2840-2862 (2016).

Singh, K.K., P.D. Shankar and K.A. Abdul: Study of microbial diversity for bacteriocin production from NGARI fish and noble synthesis of silver nanoparticles and its antimicrobial activities. World J. Pharm. Pharm. Sci., 5, 953-974 (2016).

Valgas, C., S.M. DeSouza, E.F.A. Smânia and A.J. Smânia: Screening methods to determine the antibacterial activity of natural products. Braz. J. Microbiol., 38, 369-380 (2007).

Violeta, O., S. Oana, C. Matilda, C.D. Maria, V. Catalina, C. Gheorghe, and C.C. Petruta: Production of biosurfactants and antifungal compounds by new strains of Bacillus spp. isolated from different sources. Rom. Biotech. Lett., 16, 84-91 (2011).

Wessel, D. and U.I. Flugge: A method for the quantitative recovery of protein in dilute solution in the presence of detergents and lipids. Ana. Bio., 138, 141-143 (1984). 